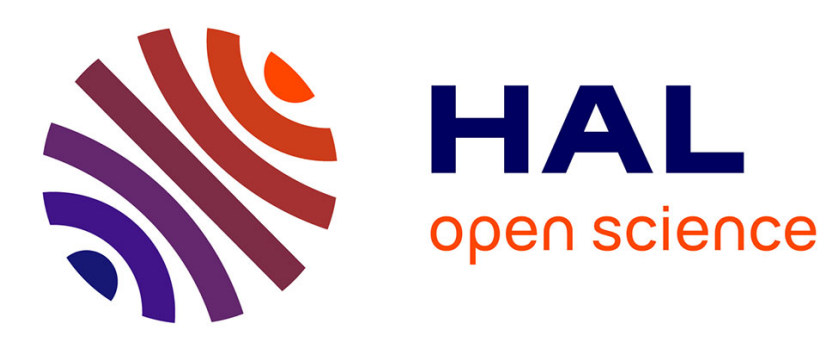

\title{
Uplink Multiple Base Stations Diversity for UNB based IoT networks
}

\author{
Yuqi Mo, Claire Goursaud, Jean-Marie Gorce
}

\section{To cite this version:}

Yuqi Mo, Claire Goursaud, Jean-Marie Gorce. Uplink Multiple Base Stations Diversity for UNB based IoT networks. CAMA 2018 - IEEE International Conference on Antenna Measurement and Applications, Sep 2018, Västerås, Sweden. pp.1-4. hal-01887640

\section{HAL Id: hal-01887640 \\ https://hal.inria.fr/hal-01887640}

Submitted on 4 Oct 2018

HAL is a multi-disciplinary open access archive for the deposit and dissemination of scientific research documents, whether they are published or not. The documents may come from teaching and research institutions in France or abroad, or from public or private research centers.
L'archive ouverte pluridisciplinaire HAL, est destinée au dépôt et à la diffusion de documents scientifiques de niveau recherche, publiés ou non, émanant des établissements d'enseignement et de recherche français ou étrangers, des laboratoires publics ou privés. 


\title{
Uplink Multiple Base Stations Diversity for UNB based IoT networks
}

\author{
Yuqi $\mathrm{MO}^{* \dagger}$, Claire GOURSAUD*, and Jean-Marie GORCE* \\ ${ }^{*}$ CITI Labs, INSA-Lyon, Villeurbanne, France \\ ${ }^{\dagger}$ Sigfox Company, Building E-volution, Labège, France \\ Email: mo.yuqi@insa-lyon.fr; claire.goursaud@insa-lyon.fr; \\ jean-marie.gorce@insa-lyon.fr;
}

\begin{abstract}
Ultra-Narrow-Band has been a promising candidate for low-power long-distance transmissions in the context of IoT. Its radio access is random at time and frequency, and can degrade the network performance. We propose to apply signal combining and interference cancellation technologies across multiple base stations in UNB networks, in order to take advantage of their spatial diversity. We evaluate and compare the performance enhancement of each technology, compared to single BS case. These technologies exploiting multi-BS diversity are proved to be significantly beneficial in improving UNB networks' scalability. We can gain until 28 times better performance with one iteration global SIC. We highlight that these results provide us a choice among the technologies according to the improvement needs and the implementation complexity.
\end{abstract}

Keywords-IoT, LPWAN, Sigfox, UNB, spectral randomness, multi-BS diversity, SC/MRC/EGC, local SIC, global SIC.

\section{INTRODUCTION}

Low Power Wide Area Network (LPWAN) has arisen as enabling technologies dedicated to low-power and longdistance transmissions in the context of IoT. Currently, the available LPWAN technologies include a large variety of alternatives such as 3GPP Narrow-Band IoT (NB-IoT), Long-Term Evolution for Machines (LTE-M), CSS (known as LoRa), UNB (known as Sigfox), and RPMA (used by Ingenu and Weightless) [1].

Among them, Ultra-Narrow-Band (UNB) developed and patented by the French company Sigfox, was the first one to be commercially initiated for IoT purpose. It is operated at the $868 \mathrm{MHz}$ (resp. $915 \mathrm{MHz}$ ) ISM band in Europe (resp. in the USA). The specificity of UNB is that the transmitted signal typically occupies $100 \mathrm{~Hz}$ [2]. This occupied bandwidth is very narrow compared to the available frequency resource, which is $192 \mathrm{kHz}$.

The radio access of UNB is RFTMA (Random Frequency Time Multiple Access), which is Aloha-based without preliminary channel sensing. Each device sends its messages at any moment and at a carrier frequency randomly chosen at its will. The advantage of this approach is the reduction of energy consumption, complexity and cost. Nonetheless, as there is no control, the network can suffer from the interference generated by simultaneous transmissions [3].

Different from traditional cellular networks, nodes are not attached to one specific base station (BS) in UNB networks, thus one transmitted packet can be perceived by several surrounding BSs. Each BS may have a different point of view of the same signal, as it experiences various channel conditions to reach each BS. Therefore, taking advantage of this multi-BS spatial diversity can be beneficial to improve the UNB system performance.

The most well-known technologies are the signal combining technologies. They are originally used in multiple-antennas system [4] where one BS combines the signals from all of its antennas (or branches) to obtain an output signal. The optimal ones are maximum ratio combining (MRC) or equal gain combining (EGC). But the receiver complexity of MRC and EGC is directly proportional to the number of branches, which makes them rarely used in real systems. A suboptimal solution is the selection combining (SC), which is simple to implement but cannot take the maximum advantage of the spatial diversity. Different from traditional multi-antennas systems, the BSs in this work have only one antenna each. Since we want to combine the signals (of the same packet) received by each BS, it can be seen as distributed multiantennas system.

The technology SIC (successive interference cancellation) allows to mitigate interference [5], and has been applied in UNB networks for single BS case [6], where the SIC gain is proved to be very limited. The very first studies, which proposed to do interference cancellation across multiple receivers, are in the context of TDMA (time division multiple access) [7] or CDMA (code division multiple access) [8]. More recent studies about multi-BS systems applying SIC are mostly focused on the slotted ALOHA scheme [9], [10].

To the best of our knowledge, there rarely exist works about applying combining technologies or SIC in distributed multi-BS systems. What's more, none of the existing studies has considered a totally random radio access scheme where the partial interference exists. Our contribution is thus to evaluate the spatial diversity gain of such combining and SIC technologies, in multi-BS systems where the nodes' carrier frequencies are random.

The rest of this paper is organized as follows: we give the network topology and hypothesis Section II. Then we propose several technologies to exploit multi-BS diversity in Section III. We give the performance evaluation of all the proposed technologies in Section IV. Finally,we conclude the paper in Section V. 


\section{Modeling And Hypothesis}

In this work, we consider the uplink of UNB networks. We assume that the nodes' distribution follows a homogeneous PPP (Poisson Point process). We focus at one observed moment. And we assume that the active nodes density $\lambda_{n}$ (nodes per $\mathrm{m}^{2}$ ) is stable at this moment. The distribution of base stations follows as well a homogeneous PPP (which is independent from the PPP of nodes), with the density $\lambda_{b}$. We define the normalized load as the ratio of active nodes and BS: $\frac{\lambda_{n}}{\lambda_{b}}$.

The behaviors of all the active nodes are assumed to be identical: they transmit with the same emission power and power gain. The nodes are not attached to any specific BSs. When active nodes broadcast their packets, they can be potentially perceived by all the BSs around. We consider the propagation path loss $\alpha$, and Rayleigh fading in this paper. Consequently, the received power of the same packet perceived by each BS can vary according to the respective channel condition (i.e. the distance and fading).

However, the SINR (signal to interference ratio) of one packet is not only impacted by the channel condition, but also the specific spectral interference of UNB. Since nodes select their carrier frequency randomly and independently, a packet may be lost (at one or several BSs) due to collisions when simultaneous transmissions happen. The spectral interference coefficient of UNB has been modeled in [3], and approximated by a zero-mean Gaussian function. It was proved to be related to the frequency spacing between nodes' carrier frequencies.

\section{TECHNOLOGIES TO EXPLOIT MULTIPLE BS SPATIAL} DIVERSITY

\section{A. Combining technologies}

To take advantage of the multi-BS spatial diversity in UNB networks, we propose to apply several technologies. The most commonly known ones are the signal combining technologies, such as selection combining (SC), max ratio combining (MRC) and equal gain combining (EGC).

As illustrated in Fig. 1, one symbol $x$ (whose amplitude can be \pm 1 ) is transmitted and perceived by all the BSs (i.e. the branches). At the $i$ th branch, its amplitude is affected by $h_{i}$ which represents the channel condition (thus the channel gain) as seen by $\mathrm{BS}_{i}$. During one transmission, the noise and interference are added, which also change the symbol's amplitude.

SC selects the best-quality signal of all the branches to determine if the issued packet can be decoded or not (i.e. if its SINR is above a threshold or not). If SC fails, the packet will be sent into MRC or EGC combiner.

As regards MRC and EGC, the combiner output is the the weighted sum of all branches' signal. The objective is to maximize the output's $\operatorname{SINR}_{y}$ by choosing the weights. In EGC, the weights for each branch are identical. In MRC, the weights are proportional to the channel gain in most of the literature where no interference is considered [4]. However, in UNB systems where the medium access is random, the interference

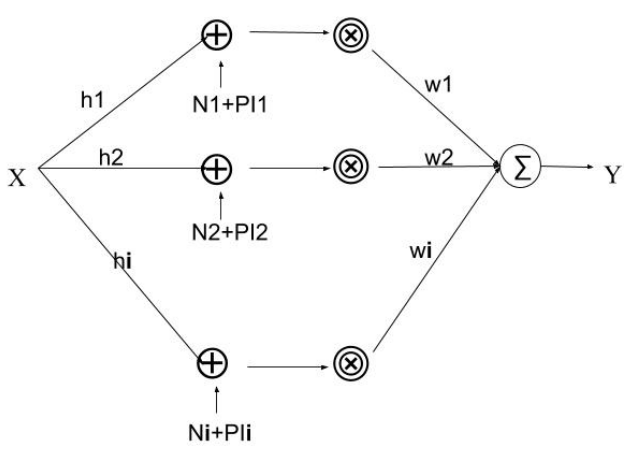

Fig. 1: Algorithm of SC/MRC/EGC. $x= \pm 1$ represents the transmitted symbol, $h_{i}$ is the channel gain of $\mathrm{BS}_{i}, N_{i}$ and $P I_{i}$ are the noise and the interference experienced by the expected signal in the branch $\mathrm{BS}_{i}, w_{i}$ is the weight at the $i$ th branch.

cannot be ignored. Consequently, when the channel gain $h_{i}$ is high in one branch, the $\operatorname{SINR}_{i}$ is not necessarily high (if it is strongly negatively affected by interference).

Therefore, different from the literature, we consider that the best-fitting branch weight $w_{i}$ is proportional to the SINR of each branch, which reflects both the channel conditions and interference. We can thus express the SINR of the MRC and EGC combiner output as the following:

$$
S I N R_{y}=\sum_{i=1}^{K} w_{i} \cdot S I N R_{i}
$$

with $K$ branches, and the weights:

$$
w_{i}(M R C)=\frac{S I N R_{i}}{\sum_{i=1}^{K} S I N R_{i}} \cdot K ; \quad w_{i}(E G C)=1
$$

\section{B. Local SIC at each BS}

To better exploit the multi-BS diversity, we consider to apply the well known technology SIC (success interference cancellation) across multiple base stations. SIC can be used to mitigate interference in random access systems [5], but mostly in single base station case. The iterative SIC procedure starts with a simple receiver (i.e. iteration 0 ), then it reconstruct and retrieve the contributions of decoded packets, which generates new decodable packets.

In this paper, we propose to perform SIC across multi-BS such that we can exploit the received power diversity and the spatial diversity jointly. We propose to do it in two ways: a local way and a global way. We assume that all the BSs can reconstruct and subtract signals perfectly, without leaving residues.

For the local SIC at each BS method, the base stations do not communicate directly with each other. They proceed SIC locally until the last iteration, then they all send their decoded packets to the back-haul, as illustrated in Fig. 2. With the presence of spatial diversity, the packets decoded in one BS may be undecodable in another BS. But since the back-haul collects the decoded packets from all the existing 


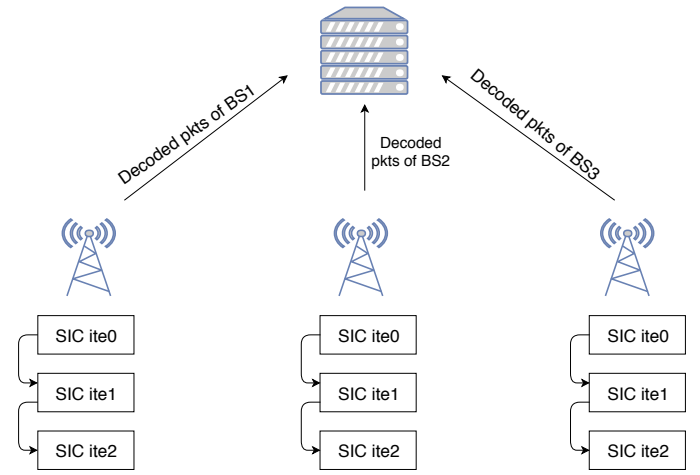

Fig. 2: Algorithm of local SIC at each BS. The BSs perform SIC locally, then forward their decoded packets to the backhaul. Ite 0 represents the procedure of a simple receiver; the maximum SIC iteration is defined to 2 .

BSs, naturally the performance is better than single BS. This procedure can be seen as a combination of selection combing across multi-BS which perform SIC locally. We note that the maximum iteration number is predefined to avoid spending too many calculation resources.

\section{Global SIC across multi-BS}

In the global case, the base stations cooperate to carry out SIC. All the BS perform SIC locally, then send their decoded packets to all the neighboring BSs directly via dedicated links, after each SIC iteration. These links are assumed to be inexpensive and provide low latency. As demonstrated in Fig. 3, the term $\mathbb{P}(i, j)$ contains the packets decoded by $B S_{i}$, after the $j$ th SIC iteration. We assume that $\mathbb{P}(i, j)$ contains all the signal details which allow other BSs to reconstruct them.

After each SIC iteration, $\mathbb{P}(i, j)$ will be broadcast to all the neighboring BSs. We assume that the base stations have the ability to relay packets for those which are physically far away, i.e. the decoded packets' exchange between $B S_{1}$ and $B S_{3}$ can be carried out by $B S_{2}$ in Fig. 3. Afterward, the BSs can exploit $\mathbb{P}(i, j)$ coming from other BSs, to reconstruct the packets they need and eliminate the issued packets' contributions. As a result, the previously interfered packets become decodable in some BSs thanks to $\mathbb{P}(i, j)$. This procedure is performed by all the BSs, after each iteration.

At the end of the last SIC iteration, all the BSs group their decoded packets through all the iterations $\operatorname{sum}(\mathbb{P}(i, j))$, then forward them to the back-haul.

\section{Performance eValuation}

In this section, we demonstrate the performance evaluation of all the technologies mentioned in the paper: SC, MRC, EGC, local SIC at each BS and global SIC across multiBS. In order to observe the gain of multiple BSs, we have also shown the performance of single BS and the nearest BS (in terms of physical distance) in addition. The term to measure the network performance is OP (outage probability), which is defined as the probability to lose a packet, i.e. the probability that the SIR of the packet is lower than the

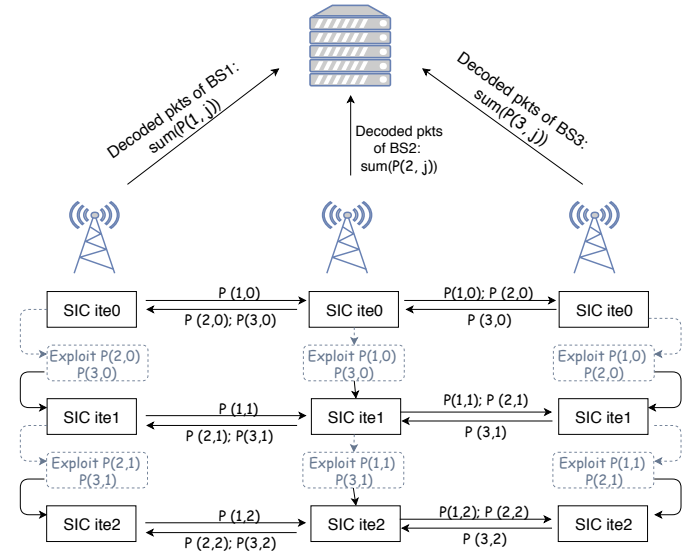

Fig. 3: Algorithm of global SIC across multiple BSs. $\mathbb{P}(i, j)$ contains the packets decoded by the $i$ th BS, after the $j$ th SIC iteration.

predefined threshold $\gamma^{*}: O P=P\left(S I R<\gamma^{*}\right)$. We have neglected the noise in this work, as its level is much lower than the interference that its ignorance does not change the network's behavior.

We have carried out Monte-Carlo simulations on matlab, with the following predefined parameters: the nodes and BSs distribution area in a $200 \mathrm{~km} \times 200 \mathrm{~km}$ square; the propagation exponent $\alpha=2$; the Rayleigh fading which follows an exponential distribution with the mean equals to 1 ; and the SIR threshold $\gamma^{*}=7 \mathrm{~dB}$. We have plotted in Fig.4 the OP with respect to the normalized load $\frac{\lambda_{n}}{\lambda_{b}}$ and the total frequency resource $B$, for all the technologies.

Firstly, we can observe that the technologies taking advantage of multi-BS have always better performance than single BS. Then we remark that $O P_{(\text {nearest } B S)}$ has worse performance than SC. This is because there are random factors such as fading, and the UNB spectral interference. Thus the shortest distance (i.e. nearest BS) does not necessarily deliver the best performance.

Secondly, we notice that MRC/EGC are always better performing than SC. This is due to the fact that the process of MRC and EGC are based on the results of selection combining. If one packet can be decoded by any one of the BSs, there is no need to send it into the MRC/EGC combiner. We only combine the packets whose $\operatorname{SIR}_{i}$ is in poor quality in all branches. Therefore, it is relevant that MRC and EGC outperform SC.

Furthermore, we remark that MRC outperforms EGC. Indeed, the weights of MRC take into account the channel gain and interference, i.e. they are proportional to the SIR of each branch. Hence the MRC weights are able to maximize the MRC combiner output's SIR. However, the EGC weights are identical in all the branches, which equalize the signals both in good and bad quality. As a consequence, even though EGC is easier to implement, its performance still falls behind MRC.

Thirdly, we can note that the global SIC across multi-BS has better performance than local SIC at each BS. This is 


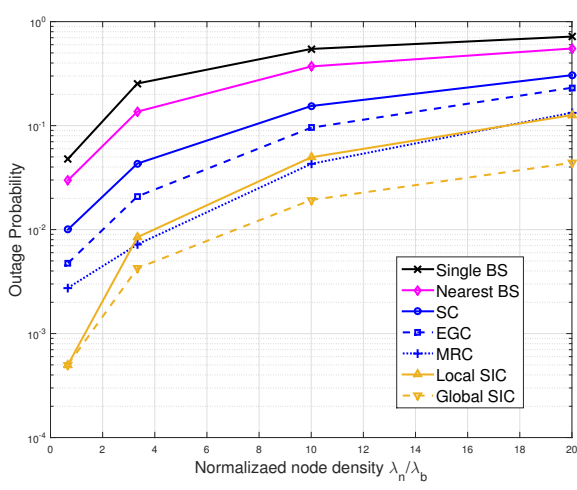

(a) OP vs $\frac{\lambda_{n}}{\lambda_{b}}$, maximum 1 SIC iteration

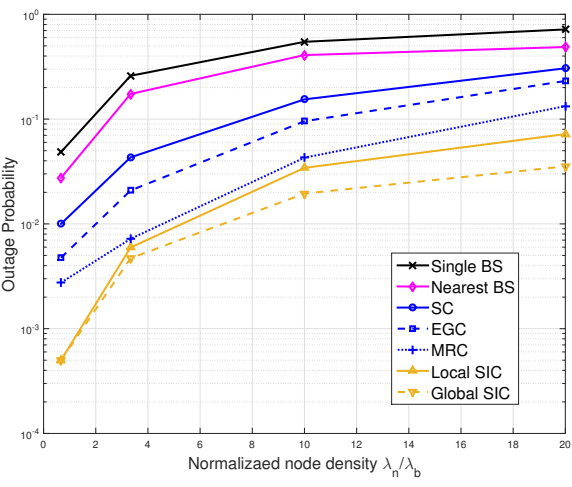

(b) OP vs $\frac{\lambda_{n}}{\lambda_{b}}$, maximum 2 SIC iteration

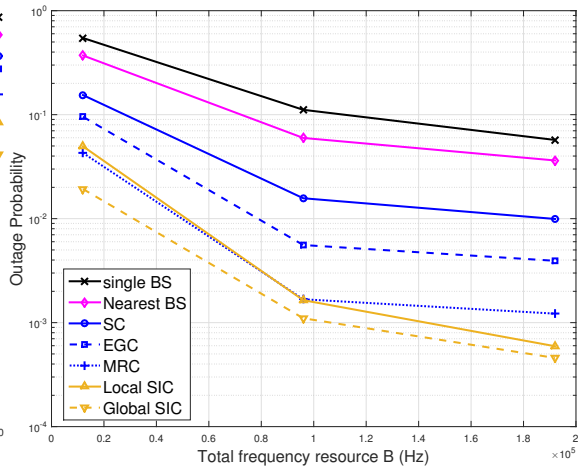

(c) OP vs $B$, maximum 1 SIC iteration

Fig. 4: (a-b) OP vs normalized load $\frac{\lambda_{n}}{\lambda_{b}}$, with $B=12 \mathrm{kHz}$; (c) OP vs total bandwidth $B$, with $\frac{\lambda_{n}}{\lambda_{b}}=10$.

not surprising since the global SIC allows all the BSs to share information with each other, and thus to reconstruct and subtract the packets decoded by other BSs. This results in decoding more packets than performing SIC locally at each BS. It is also the reason why global SIC outperforms MRC, as MRC adds up both the signals and the interferences, while global SIC can retrieve interferences.

Interestingly, when we compare Fig.4 $(a, b)$, we remark that local and global SIC only have slight performance enhancement when the maximum iteration number increases. Moreover, the higher the SIC iteration becomes, the higher the system complexity becomes, and the more computation resource we need. Thus through this result, we know that we can gain almost the same with 1 SIC iterations, without spending more computation resource to increase it to 2 .

Finally, we report the multi-BS diversity gain when compared to single BS case: $\frac{O P_{(\text {singleBS }}}{\left.O P_{(\text {multiBS }}\right)}$, as well as the implementation complexity of each technology in Table I. We note that these results give us a choice among these technologies, depending on the performance improvement we need, the budgets we have, and the infrastructure complexity we expect. For example, if we intended to implement local SIC at each BS with no more than 1 iteration (maybe due to the computation limitations), now we know that MRC can also meet our needs as they deliver similar performance. We can thus choose MRC rather than local SIC, which is less complex to implement without loss of performance.

\section{CONCLUSION}

In this paper, we have exploited the multi-BS diversity in UNB networks whose performance suffers from its random radio access. We have proposed to apply signal combining and local/global interference cancellation in the multi-BS systems. We have shown that the global SIC has the best performance, whereas it has also the highest complexity to implement. The suboptimal ones are MRC and local SIC which deliver similar enhancement, and are less complex. We highlight that the results provide a global view of the mentioned technologies' diversity gain and implementation complexity.
TABLE I: Multi-BS diversity gain compared to single BS (with $\frac{\lambda_{n}}{\lambda_{b}}=10, B=12 \mathrm{kHz}$, maximum 1 SIC iterations), and implementation complexity.

\begin{tabular}{|l|c|c|c|c|c|c|}
\hline & Nearest BS & SC & EGC & MRC & $\begin{array}{l}\text { local } \\
\text { SIC }\end{array}$ & $\begin{array}{l}\text { global } \\
\text { SIC }\end{array}$ \\
\hline Multi-BS gain & 1.5 & 4 & 6 & 12 & 11 & 28 \\
\hline Complexity & $*$ & $*$ & $* *$ & $* * *$ & $* * * *$ & $* * * * *$ \\
\hline
\end{tabular}

\section{ACKNOWLEDGMENT}

The authors would like to thank Christophe Fourtet (Sigfox company) for providing realistic UNB network parameters in this study.

\section{REFERENCES}

[1] U. Raza, P. Kulkarni, and M. Sooriyabandara, "Low power wide area networks: An overview," IEEE Communications Surveys Tutorials, vol. 19, no. 2, pp. 855-873, Secondquarter 2017.

[2] http://www.sigfox.com/en/ \\#!/technology, accessed: 2018-05-09.

[3] M.-T. Do, C. Goursaud, and J.-M. Gorce, "Interference modelling and analysis of random fdma scheme in ultra narrowband networks," in AICT 2014, 2014.

[4] A. Goldsmith, Wireless communications. Cambridge university press, 2005.

[5] S. Sen, N. Santhapuri, R. R. Choudhury, and S. Nelakuditi, "Successive interference cancellation: A back-of-the-envelope perspective," in Proceedings of the 9th ACM SIGCOMM Workshop on Hot Topics in Networks, ser. Hotnets-IX. New York, NY, USA: ACM, 2010, pp. $17: 1-17: 6$

[6] Y. Mo, C. Goursaud, and J. M. Gorce, "On the benefits of successive interference cancellation for ultra narrow band networks : Theory and application to iot," in 2017 IEEE International Conference on Communications (ICC), May 2017, pp. 1-6.

[7] E. Aktas, J. Evans, and S. Hanly, "Distributed decoding in a cellular multiple-access channel," in International Symposium onInformation Theory, 2004. ISIT 2004. Proceedings., June 2004, pp. 484-.

[8] D. Gesbert, S. Hanly, H. Huang, S. S. Shitz, O. Simeone, and W. Yu, "Multi-cell mimo cooperative networks: A new look at interference," IEEE Journal on Selected Areas in Communications, vol. 28, no. 9, pp. 1380-1408, December 2010.

[9] D. Jakoveti, D. Bajovi, D. Vukobratovi, and V. Crnojevi, "Cooperative slotted aloha for multi-base station systems," IEEE Transactions on Communications, vol. 63, no. 4, pp. 1443-1456, Apr. 2015.

[10] A. Munari, F. Clazzer, and G. Liva, "Multi-receiver aloha systems a survey and new results," in 2015 IEEE International Conference on Communication Workshop (ICCW), Jun. 2015, pp. 2108-2114. 